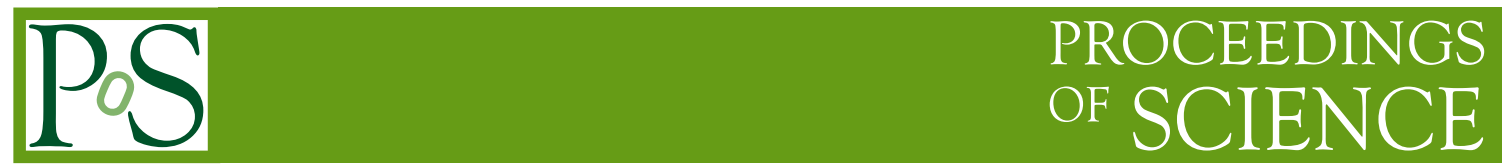

\title{
Metadynamics Remedies for Topological Freezing
}

\section{Francesco Sanfilippo*}

INFN, Sezione di Roma Tre, Via della Vasca Navale 84, I-00146 Rome, Italy

E-mail: francesco.sanfilippoeroma3.infn.it

\section{A. Laio}

SISSA, Via Bonomea 265, I-34136, Trieste

\section{G. Martinelli}

Universita di Roma La Sapienza and INFN, Piazzale Aldo Moro 5, 00185 Roma, Italy

In this presentation we show that metadynamics, when used to simulate $C P^{N-1}$, allows to address efficiently of freezing of topological charge, to reconstruct the free energy of the topological charge $F(Q)$ and to compute the topological susceptibility as a function of the coupling and of the volume. We discuss possible extensions to QCD.

34th annual International Symposium on Lattice Field Theory

24-30 July 2016

University of Southampton, UK

${ }^{*}$ Speaker. 


\section{Introduction}

In the recent literature the problem of very long autocorrelation times and of the theoretical control over the systematic error in numerical simulations has been addressed in a series of papers $[1,2,3,4,5,6,7,8]$ and several solutions to the topological critical slowing down have been proposed $[9,10,11,12,13,14,15,16,17]$.

Here we propose to address the problem of topological trapping by using metadynamics, that was introduced to enhance the probability of observing rare conformational changes and reconstructing the free energy in biophysics, chemistry and material sciences systems [18, 19].

In metadynamics, the dynamics in the space of a set of Collective Variables (CVs) is enhanced by a history-dependent potential constructed as a sum of Gaussians centered along the trajectory followed by the CVs. The sum of Gaussians is exploited to reconstruct iteratively an estimator of the free energy. The system has access to a feedback which during the time evolution fills the local free energy minima. Thus, even if at the beginning the system visits more often the region at the bottom of a local minimum, after a few steps it starts exploring regions corresponding to higher and higher values of the free energy. Sooner or later, the system fills the minimum, climbs out of it, and visits another minimum that is eventually also filled, until all the relevant minima are explored. The key idea of metadynamics is exploiting the time-dependent bias potential itself as a free energy estimator. In particular, the time average of the bias potential has been shown to converge to the negative of $F$ with an error that scales to zero with the inverse square root of the simulation time [20].

Here we propose to address the problem of topological trapping by performing metadynamics on the topological charge. We will show that this approach induces a large number of transitions between different sectors, and therefore converges very rapidly. At the same time, the approach allows computing the unbiased average value of any observable by standard reweighting techniques. In order to test our proposal we first study the two-dimensional $C P^{N-1}$ models that have several features in common with $Q C D$, such as asymptotic freedom and a non-trivial topological structure. Since these models require much smaller computing resources, they are an ideal theoretical laboratory to be used in an early and exploratory stage of any new algorithm. We find the improvement induced by metadynamics considerable and worth to be implemented in a $Q C D$ study that we plan to perform in the near future. This presentation is based on our recent paper [21] to which we refer for more details.

\section{2. $C P^{N-1}$ and the different definitions of the topological charge on the lattice}

In the continuum the two dimensional $C P^{N-1}$ model is defined by the action

$$
S=\frac{1}{g} \int d^{2} x \bar{D}_{\mu} \bar{z} D_{\mu} z
$$

where $z$ is a complex $N$-dimensional field with unit norm $\bar{z} \cdot z=\sum_{i=1}^{N} z_{i}^{*} z_{i}=1$ and the covariant derivative is given by $D_{\mu}=\partial_{\mu}+i A_{\mu}$. The connected correlation function is defined as

$$
G(x)=\langle\operatorname{Tr} P(x) P(0)\rangle-\frac{1}{N}\langle\operatorname{Tr} P(x)\rangle\langle\operatorname{Tr} P(0)\rangle, \quad P(x) \equiv \bar{z}(x) \otimes z(x) \quad\left(P_{i, j}(x)=z_{i}^{*} z_{j}\right)
$$


The topological-charge density $q(x)$, the total charge $Q$ and its susceptibility $\chi_{t}$ are defined as

$$
q(x)=\frac{1}{2 \pi} \varepsilon_{\mu v} \partial_{\mu} A_{v}, \quad Q=\int d^{2} x q(x), \quad \chi_{t}=\int d^{2} x\langle q(x) q(0)\rangle=\frac{\left\langle Q^{2}\right\rangle}{V} .
$$

The lattice action is given by [22] $S=\frac{1}{g} \sum_{\vec{n}, \mu} \bar{D}_{\mu} \bar{z}_{\vec{n}} D_{\mu} z_{\vec{n}}$, where we introduce the lattice covariant derivative $D_{\mu} \bar{z}_{\vec{n}}=\lambda_{\vec{n}, \hat{\mu}} \bar{z}_{\vec{n}+\hat{\mu}}-\bar{z}_{\vec{n}}$, expressed in term of the $U(1)$ gauge link $\lambda_{\vec{n}, \hat{\mu}} \equiv \exp \left(i A_{\mu}(\vec{n}+\right.$ $\hat{\mu} a / 2)$, where $a$ is the lattice spacing and $\hat{\mu}$ the unit vector in the direction $\mu$. We use the lattice definition of the correlation length $\xi_{G}[2]$

$$
\xi_{G}^{2}=\frac{1}{4 \sin ^{2}\left(q_{m} / 2\right)} \frac{\tilde{G}(0)-\tilde{G}\left(q_{m}\right)}{\tilde{G}\left(q_{m}\right)},
$$

where $q_{m}$ is the smallest non zero dimensionless momentum on a lattice with lattice spacing $a$ and physical volume $a L$, namely $q_{m}=(2 \pi / L, 0)$.

A definition of the topological charge that serves our scope is given in terms of the imaginary part of the plaquette, as illustrated in Ref. [23]

$$
Q^{\lambda}=\frac{1}{2 \pi} \sum_{n} \mathfrak{I}\left[\lambda_{\mu}(n) \lambda_{v}(n+\hat{\mu}) \bar{\lambda}_{\mu}(n+\hat{v}) \bar{\lambda}_{v}(n)\right] \quad \mu<v .
$$

We make use of a modification of the stout smearing [24], adapted to treat $U(1)$ variables to reduce the noise associated with such definition.

\section{Metadynamics}

In metadynamics the action $S(x)$ is modified by adding to it a history-dependent potential

$$
V_{G}(Q(x), t)=\sum_{t^{\prime}=\tau_{G}, 2 \tau_{G}, \ldots, t^{\prime}<t} g\left(Q(x)-q\left(t^{\prime}\right)\right)
$$

where $g(q)$ is a non-negative function of its argument, that rapidly vanishes for large $|q|$. In the original implementation, $g(q)=w \exp \left(-\frac{q^{2}}{2 \delta q^{2}}\right)$, where $w$ and $\delta q$ are two parameters that can be used to tune the growth speed of $V_{G}$. Thus, the metadynamics potential is a sum of small repulsive potentials, acting on the $\mathrm{CV}$, and localized on all the configurations $q(t)$ that have already been explored during the trajectory, up to time $t$. This potential disfavors the system from revisiting configurations that have already been visited. If the dynamics of $q$ is bound in a finite connected region, after a transient the probability distribution of $q$ in this region can only be approximately flat. Indeed, if this is not the case, by definition the system would spend more time in a subregion of $q$, and $V_{G}$ would grow preferentially in that region, disfavoring the system from remaining there. Thus, deviations from the flat distribution can only survive for a transient time. $P(q) \exp \left(-V_{G}(q, t)\right)$ must be approximately constant or, equivalently,

$$
V_{G}(q, t) \sim-F(q) .
$$

This equation states that in metadynamics the free energy is estimated by the negative of the bias potential itself. More precisely, since eq. (3.2) is valid at any time, the best estimator of the free energy at time $t$ is given by the (large) time average of $V_{G}$ up to time $t$,

$$
-F(q) \sim \overline{V_{G}(q, t)}=\frac{1}{t-t_{e q}} \int_{t_{e q}}^{t} d t^{\prime} V_{G}\left(q, t^{\prime}\right)
$$


The equilibration time $t_{e q}$ entering in eq. (3.3) is the time at which the history dependent potential becomes approximately stationary (or, equivalently, the probability distribution as a function of $q$ becomes approximately flat). Like in the ordinary estimates of the average value of an observable, the exact choice of $t_{e q}$ influences only the convergence speed, but not the final result. The difference between $-F$ and $\overline{V_{G}}$ in eq. (3.3) decreases as the square root of $t-t_{e q}$, with a prefactor that strongly depends on the specific $\mathrm{CV} q$ [20].

For the sake of computational efficiency, we store the history-dependent potential on a regular grid of spacing $\delta q ;\left(q_{0}, q_{1}, \cdots, q_{n}\right)$, with $q_{i}=q_{0}+i \delta q$. The use of the grid makes it possible to carry on metadynamics for long runs at a fixed overhead per sweep (in computer time), whereas the computer time with the naive procedure would linearly increase with the number of sweeps.

At the beginning of the simulation we set $V_{i}=V_{G}\left(q_{i}\right)=0$. Then, at every step, we

1. compute the value of the $\mathrm{CV} q(t) \equiv Q^{\lambda}(t)$;

2. find the grid interval $i$ where it falls $i=\operatorname{int}\left(\frac{q(t)-q_{0}}{\delta q}+0.5\right)$;

3. update the potential, $V_{i}=V_{i}+w\left(1-\frac{q(t)-q_{i}}{\delta q}\right), \quad V_{i+1}=V_{i+1}+w \frac{q(t)-q_{i}}{\delta q}$.

The force ruling the evolution of the fields $x$ is then changed by adding to it the component deriving from the history-dependent potential. The optimal grid spacing $\delta q$ must be such that i) the potential wells are filled rapidly, and this requires a large $\delta q$; ii) the free energy $F(q)$, eq. (3.2), can be accurately reconstructed.

The unbiased expectation value of an observable $O$ is computed through reweighting

$$
\langle O\rangle=\frac{\sum_{i} O_{i} \exp \left(-\overline{V\left(Q_{i}^{\lambda}\right)}\right)}{\sum_{i} \exp \left(-\overline{V\left(Q_{i}^{\lambda}\right)}\right)},
$$

where $\left.\overline{V\left(Q^{\lambda}\right)}\right)$ is defined in eq. (3.3).

\section{Numerical results}

We have studied $C P^{N-1}$, with $N=21$, at several values of the coupling constant, with different physical volumes at fixed correlation length and with different values of the correlation length at fixed physical size.

\subsection{A comparison of standard HMC and metadynamics}

We start by considering metadynamics run in the region of parameters where the standard HMC is still able to explore different topological sectors, and achieve convergence. This allows checking if the two approaches provide consistent results.

The most important effect of the metadynamics bias is reducing the autocorrelation time of the observables by orders of magnitude. For example in the case $\beta=0.75$ and $L=60$ (already explored in Ref. [23]) we find $\tau_{Q}=155000 \pm 10000$ in the HMC run, whereas the corresponding value for the metadynamics run is $\tau_{Q}=5600 \pm 1000$. A related quantity is the transition probability per unit time between two different topological sectors, $v$. This quantity is defined as the number 

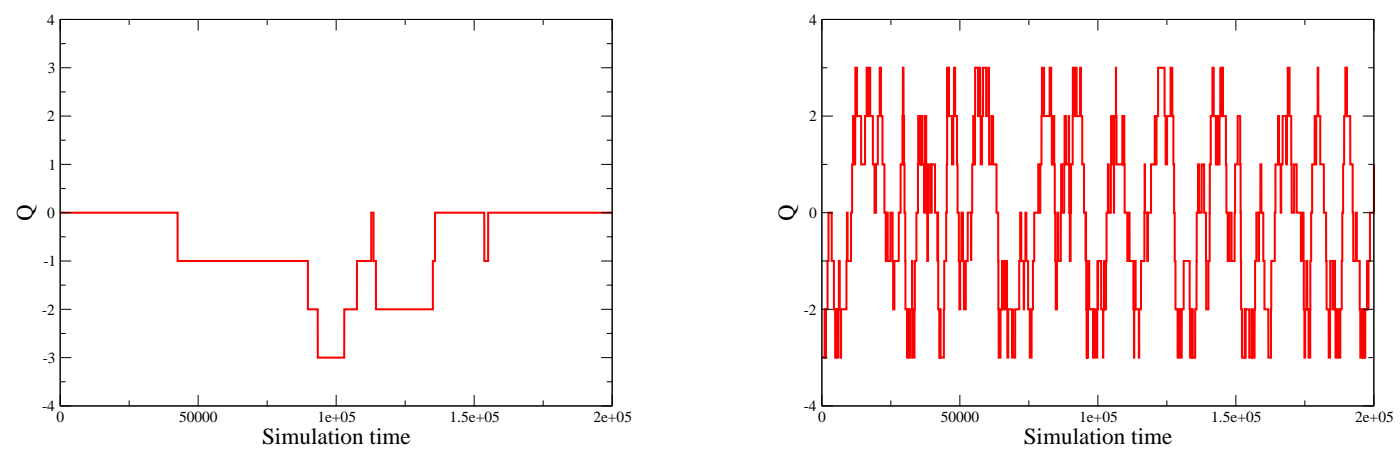

Figure 1: $(a)$ and (b) Topological charge as a function of the number of sweeps with the standard HMC and metadynamics respectively at $\beta=0.75$ and $L=60$.

of jumps between two different values of $Q^{g}$ divided by the total number of sweeps. For the two runs corresponding to Fig. 1, we have $v=4.98 \cdot 10^{-5}$ with the $\mathrm{HMC}$ and $v=2.24 \cdot 10^{-3}$ for metadynamics respectively.

One of the main results of the method is the reconstructed free energy. In Fig. 2 we compared the reconstructed average free energy of the topological charge $F\left(Q^{\lambda}\right)$ obtained with metadynamics, with $-\log \left(P\left(Q^{\lambda}\right)\right)$ estimated in a standard HMC run. Remarkably, the two estimates are fully consistent within the small error bars, indicating that metadynamics allows computing reliably the probability distribution of the charge. $F(Q)$ is very well approximated by the function

$$
F(Q)=A Q^{2}+B \sin ^{2}(\pi Q)
$$

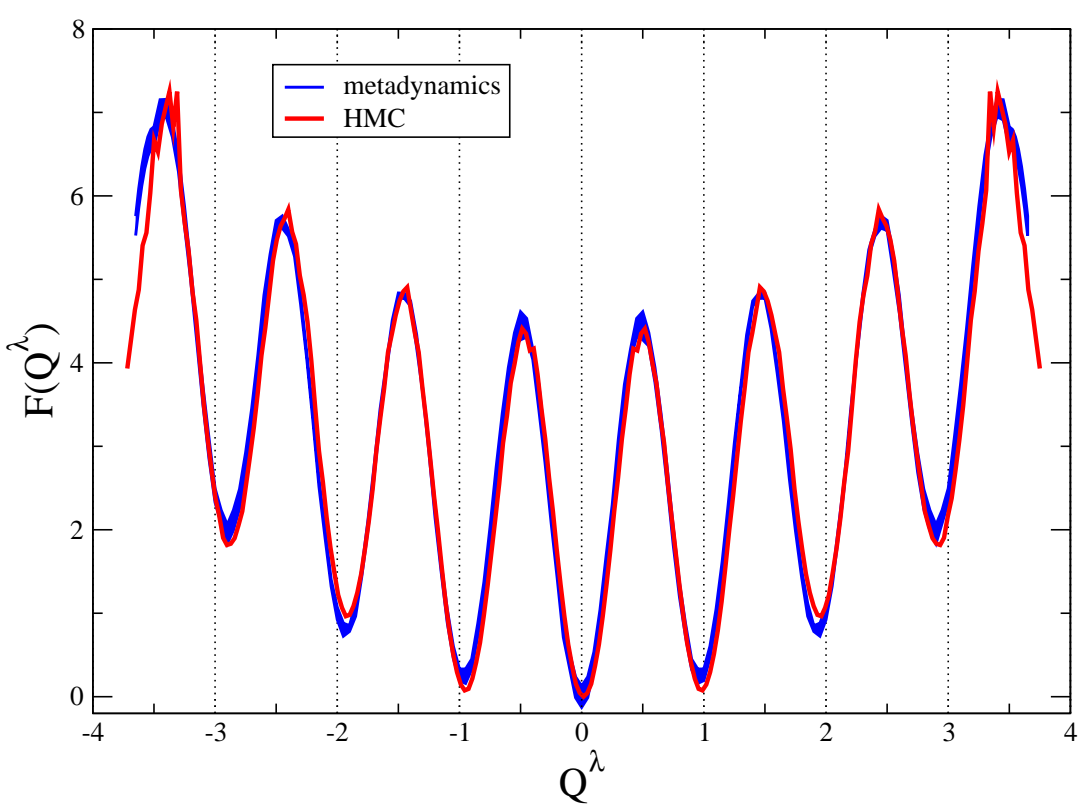

Figure 2: Comparison between the free energy $F\left(Q^{\lambda}\right)$ estimated by 30k sweeps with metadynamics and $-\log (P(Q))$ estimated wiht 300k sweeps of standard HMC at $\beta=0.70$ with $L=62$. 
where $A$ and $b$ are numerical constants which in general, at fixed $N$, depend on $L$ and $\beta$.

As an example, in Fig. 2 we fitted the effective potential reported in the metadynamics case, obtaining $A=0.20 \pm 0.06$ and $B=4.38 \pm 0.10$, with a $\chi^{2} /$ dof $\sim 1$. By using the relation $A \sim$ $1 /\left(2 \chi_{t} V\right)$, this corresponds to $\chi_{t}=(6.50 \pm 1.9) \times 10^{-4}$ well compatible with the results known in the literature for this setup (see Ref. [23]).

\subsection{The central question: Towards the continuum limit}

In order to demonstrate that the approach presented in this work allows addressing efficiently the problem of topological trapping, we now discuss the scaling of the autocorrelation time as a function of the lattice spacing $a \sim \xi_{G}^{-1}$, namely of $\xi_{G}$, and of the physical volume $L / \xi_{G}$. In the left panel of Fig. 3 we display the dependence of $v$ on $\xi_{G}$ with HMC and metadynamics at several values of $L / \xi_{G}$. As expected, because of entropy, in the standard HMC $v$ is an increasing function of the lattice size, and this corresponds to an increase in the dispersion of the topological charge, namely of $\left\langle Q^{2}\right\rangle$. At larger values of $\beta$, as we proceed toward the continuum limit, with the standard HMC, $v$ decreases exponentially as a function of the correlation length $\xi_{G}[1,2]$, so that for $a \xi_{G} \gtrsim 9$ the changes of the topological sector are so rare that only an upper limit for $v$ can be provided.

The behavior observed with the HMC is to be contrasted with the results obtained with metadynamics (corrected for the bias using eq. 3.4) since in this case $v$ is sensibly flatter, and the simulation spans all the possible sectors of $Q$ allowing to produce reliable results.

To assess the scaling of error on observable quantity estimated with Metadynamics, in Fig. 3 we compare the Renormalization Group Invariant quantity $\xi_{G}^{2} \chi_{Q}$ estimated with Metadynamics and Hybrid Monte Carlo, for a fixed number of sweeps. At coarse lattice spacing (small values of $\xi_{G} / a$ ) the two estimate are both accurate and in agreement with each other. Increasing the lattices psacing (moving to larger values of $\xi_{G} / a$ ) it is clear that the HMC estimate deteriorate very quickly, reaching soon a point where only an upper estimate can be given. On the contrary, the error of Metadynamics estimate grows mildly with the decrease of the lattice spacing, and the various estimates remains consistent with each others.
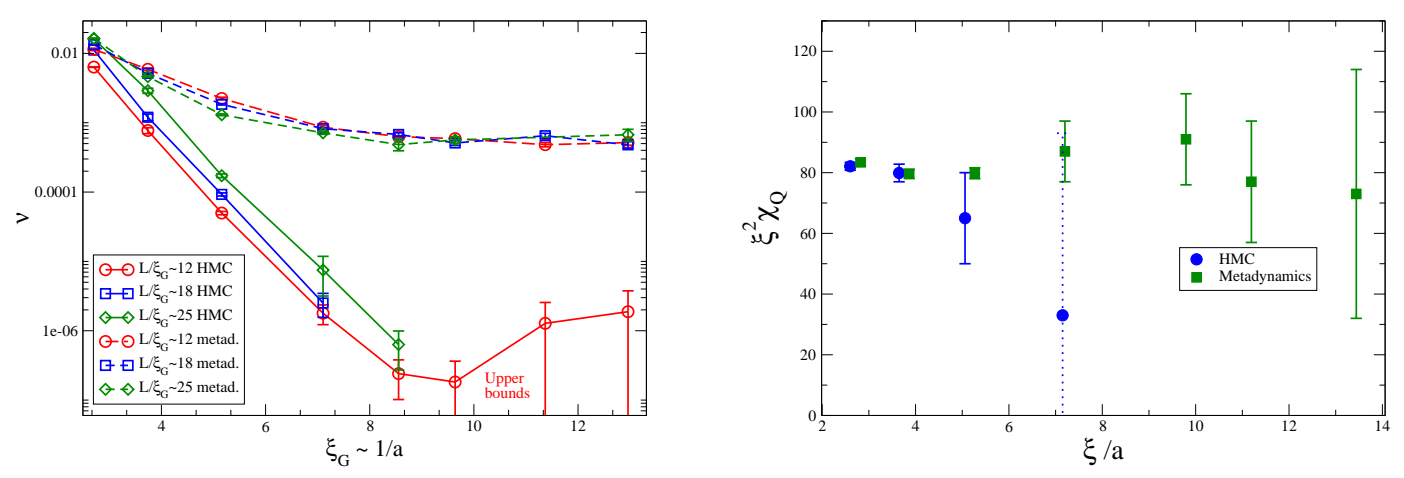

Figure 3: Left: Scaling of the frequency $v$ as a function of $\xi_{G}$ with metadynamics (dashed) and HMC (full line). Right: $\xi_{G}^{2} \chi_{Q}$ estimated with Metadynamics and Hybrid Monte Carlo, for a fixed number of sweeps, for a number of lattice spacing. 


\section{Conclusions}

We have shown that the metadynamics approach $[18,19]$ can be used to simulate $C P^{N-1}$ improving dramatically the problem of the slowing down observed in numerical simulations for quantities related to the topological charge.

The much reduced slowing down allows us to study a range of $\beta$ much larger than that available with ordinary HMC. It seems straightforward to extend the general method exposed to the case of QCD. Metadynamics might also offer a solution to the simulating theories with complex actions (for example QCD with a non zero $\theta$ term or at finite chemical potential).

\section{References}

[1] L. Del Debbio, H. Panagopoulos and E. Vicari, JHEP 0208 (2002) 044 [hep-th/0204125].

[2] L. Del Debbio, G. M. Manca and E. Vicari, Phys. Lett. B 594 (2004) 315 [hep-lat/0403001].

[3] S. Schaefer et al. [ALPHA Collaboration], Nucl. Phys. B 845 (2011) 93 [arXiv:1009.5228 [hep-lat]].

[4] G. P. Engel and S. Schaefer, Comput. Phys. Commun. 182 (2011) 2107 [arXiv:1102.1852 [hep-lat]].

[5] A. Chowdhury et al., J. Maiti and P. Majumdar, JHEP 1402 (2014) 045 [arXiv:1311.6599 [hep-lat]].

[6] R. C. Brower et al., Phys. Rev. D 90 (2014) 1, 014503 [arXiv:1403.2761 [hep-lat]].

[7] J. Flynn, A. Juttner, A. Lawson and F. Sanfilippo, arXiv:1504.06292 [hep-lat].

[8] M. Cè et al., Phys. Rev. D 92, no. 7, 074502 (2015) [arXiv:1506.06052 [hep-lat]].

[9] P. de Forcrand et al., Nucl. Phys. Proc. Suppl. 63 (1998) 679 [hep-lat/9709104].

[10] M. Luscher, PoS LATTICE 2010 (2010) 015 [arXiv:1009.5877 [hep-lat]].

[11] M. Luscher and S. Schaefer, JHEP 1107 (2011) 036 [arXiv:1105.4749 [hep-lat]].

[12] A. Ramos, PoS LATTICE 2012, 193 (2012) [arXiv:1212.3800 [hep-lat]].

[13] G. McGlynn and R. D. Mawhinney, PoS Lattice 2013, 027 (2014) [arXiv:1311.3695 [hep-lat]].

[14] U. Gerber et al., PoS LATTICE 2014, 320 (2014) [arXiv:1410.0426 [hep-lat]].

[15] M. G. Endres et al., arXiv:1510.04675 [hep-lat].

[16] A. S. Gambhir and K. Orginos, PoS LATTICE 2014, 043 (2015) [arXiv:1506.06118 [hep-lat]].

[17] A. Dromard et al., arXiv:1510.08809 [hep-lat].

[18] A. Laio, M. Parrinello, Proceedings of the National Academy of Sciences, 2002, 99.20: 12562-12566.

[19] A. Laio and F.L. Gervasio, Rep. Prog. Phys. 71, 126601, 2008

[20] Y. Crespo, F. Marinelli, F. Pietrucci, A. Laio, Physical Review E, 81, 055701 (2010).

[21] A. Laio, G. Martinelli and F. Sanfilippo, JHEP 1607 (2016) 089.

[22] P. Di Vecchia, A. Holtkamp, R. Musto, F. Nicodemi and R. Pettorino, Nucl. Phys. B 190 (1981) 719.

[23] M. Campostrini, P. Rossi and E. Vicari, Phys. Rev. D 46 (1992) 2647.

[24] C. Morningstar and M. J. Peardon, Phys. Rev. D 69 (2004) 054501 [hep-lat/0311018]. 